\title{
Working modes and aspects in fully parallel manipulators
}

\author{
Damien Chablat \\ Philippe Wenger \\ Institut de Recherche en Cybernétique de Nantes \\ École Centrale de Nantes \\ 1, rue de la Noë, 44321 Nantes, France \\ Damien.Chablat@lan.ec-nantes.fr Philippe.Wenger@lan.ec-nantes.fr
}

\begin{abstract}
The aim of this paper is to characterize the notion of aspect in the workspace and in the joint space for parallel manipulators. In opposite to the serial manipulators, the parallel manipulators can admit not only multiple inverse kinematic solutions, but also multiple direct kinematic solutions. The notion of aspect introduced for serial manipulators in [1], and redefined for parallel manipulators with only one inverse kinematic solution in [4], is redefined for general fully parallel manipulators. Two Jacobian matrices appear in the kinematic relations between the joint-rate and the Cartesian-velocity vectors, which are called the "inverse kinematics" and the "direct kinematics" matrices. The study of these matrices allow to respectively define the parallel and the serial singularities. The notion of working modes is introduced to separate inverse kinematic solutions. Thus, we can find out domains of the workspace and the joint space exempt of singularity. Application of this study is the moveability analysis in the workspace of the manipulator as well as path-planing and control. This study is illustrated in this paper with a RR-RRR planar parallel manipulator.

KEY WORDS : Kinematics, Fully Parallel Manipulator, Aspects, Working modes, Singularity.
\end{abstract}

\section{Introduction}

A well known feature of parallel manipulators is the existence of multiple solutions to the direct kinematic problem. That is, the mobile platform can admit several positions and orientations (or configurations) in the workspace for one given set of input joint values [3]. Moreover, parallel manipulators exist with multiple inverse kinematic solutions. This means that the mobile platform can admit several input joint values corresponding to one given configuration of the end- effector. To cope with the existence of multiple inverse kinematic solutions in serial manipulators, the notion of aspects was introduced in [1]. The aspects equal the maximal singularity-free domains in the joint space. For usual industrial serial manipulators, the aspects were found to be the maximal sets in the joint space where there is only one inverse kinematic solution.

A definition of the notion of aspect was given by 2 for parallel manipulators with only one inverse kinematic solution. These aspects were defined as the maximal singularity-free domains in the workspace. For instance, this definition can apply to the Stewart platform [4.

First of all, the working modes are introduced to allow the separation of the inverse kinematic solutions. Then, a general definition of the notion of aspect is given for all fully parallel manipulators. The new aspects are the maximal singularity-free domains of the Cartesian product of the workspace with the joint space.

A possible use of these aspects are the determination of the best working mode. It allows to achieve complex task in the workspace or to make pathplaning without collision. As a matter of fact, currently, the parallel manipulators possessing multiple inverse kinematic solutions evolve only in one working mode. For a given working mode, the aspect associated is different. It is possible to choose one or several working modes to execute the tasks expected in the maximal workspace of the manipulator.

\section{Preliminaries}

In this paragraph, some definitions permitting to introduce the general notion of aspect are quoted.

\subsection{The fully parallel manipulators}

Definition 1 A fully parallel manipulator is a mechanism that includes as many elementary kinematic 
chains as the mobile platform does admit degrees of freedom. Moreover, every elementary kinematic chain possesses only one actuated joint (prismatic, pivot or kneecap). Besides, no segment of an elementary kinematic chain can be linked to more than two bodies [3].

In this study, kinematic chains are always independent. This condition is necessary to find the working modes. Also, the elementary kinematic chains can be called "legs of the manipulator" [5].

\section{$2.2 \quad$ Kinematic relations}

For a manipulator, the relation permitting the connection of input values $(\mathbf{q})$ with output values $(\mathbf{X})$ is the following

$$
F(\mathbf{X}, \mathbf{q})=0
$$

This definition can be applied to serial or parallel manipulators. Differentiating equation (11) with respect to time leads to the velocity model

$$
\text { At }+\mathbf{B} \dot{\mathbf{q}}=0
$$

With

$$
\begin{aligned}
& \mathbf{t}=\left[\begin{array}{c}
w \\
\dot{\mathbf{c}}
\end{array}\right] \text { For planar manipulators. } \\
& \mathbf{t}=\left[\begin{array}{c}
\mathbf{w}
\end{array}\right] \text { For spherical manipulators } . \\
& \mathbf{t}=\left[\begin{array}{c}
\mathbf{w} \\
\dot{\mathbf{c}}
\end{array}\right] \text { For spatial manipulators. }
\end{aligned}
$$

Where $w$ is the scalar angular-velocity and $\dot{\mathbf{c}}$ is the two-dimensional velocity vector of the operational point of the moving platform for the planar manipulator. For the spherical and the spatial manipulator, $\mathbf{w}$ is the three-dimensional angular velocity-vector of the moving platform. And $\dot{\mathbf{c}}$ is the three-dimensional velocity vector of the operational point of the moving platform for the spatial manipulator.

Moreover, $\mathbf{A}$ and $\mathbf{B}$ are respectively the directkinematics and the inverse-kinematics matrices of the manipulator. A singularity occurs whenever A or B, (or both) that can no longer be inverted. Three types of singularities exist [6]:

$$
\begin{aligned}
\operatorname{det}(\mathbf{A}) & =0 \\
\operatorname{det}(\mathbf{B}) & =0 \\
\operatorname{det}(\mathbf{A}) & =0 \text { and } \operatorname{det}(\mathbf{B})=0
\end{aligned}
$$

\subsection{Parallel singularities}

Parallel singularities occur when the determinant of the direct kinematics matrix $\mathbf{A}$ vanishes. The corresponding singular configurations are located inside the workspace. They are particularly undesirable because the manipulator can not resist any force and control is lost.

\subsection{Serial singularities}

Serial singularities occur when the determinant of the inverse kinematics matrix $\mathbf{B}$ vanishes. When the manipulator is in such a singularity, there is a direction along which no Cartesian velocity can be produced.

\subsection{Postures and assembling modes}

The multiple inverse kinematic solutions induce multiple postures for each leg.

Definition $2 A$ posture changing trajectory is equivalent to a trajectory between two inverse kinematic solutions.

The multiple direct kinematic solutions induce multiple assembling modes for the mobile platform.

Definition $3 A n$ assembling mode changing trajectory is equivalent to a trajectory between two direct kinematic solutions.

As an example, the 3-RRR planar parallel manipulator (the first joints are actuated joints), a posture changing trajectory exists between two inverse kinematic solutions (Fig. 11) and an assembling mode trajectory exits between two direct kinematic solutions (Fig. 2). In these trajectories, the mobile platform can meet a singular configuration.

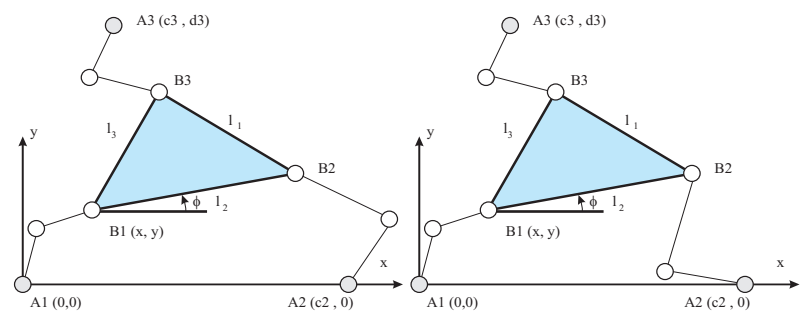

Figure 1: Two postures

\subsection{Working Modes}

The working modes are defined for fully parallel manipulators (Def. 11). From this definition, the inversekinematic matrix is always diagonal. For a manipulator with $n$ degrees of freedom, the inverse kinematic matrix $\mathbf{B}$ is like in eq. (3). Each term $\mathbf{B}_{j j}$ is associated 


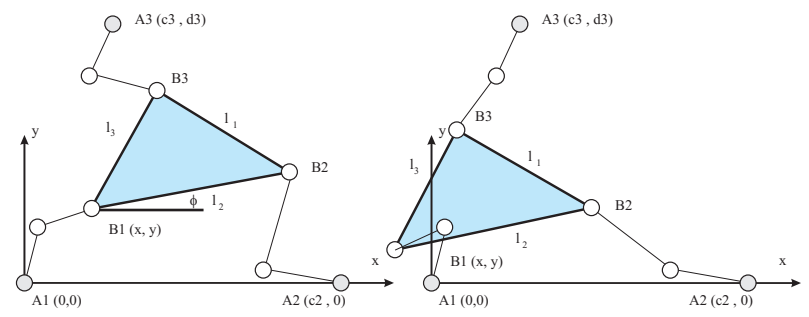

Figure 2: Two assembling modes

with one leg. Its vanishing induces the apparition of a serial singularity.

$$
\mathbf{B}=\left[\begin{array}{ccccc}
\mathbf{B}_{11} & 0 & \cdots & \cdots & 0 \\
0 & \ddots & & \ddots & \vdots \\
\vdots & & \mathbf{B}_{j j} & & \vdots \\
\vdots & \ddots & & \ddots & 0 \\
0 & \cdots & \ldots & 0 & \mathbf{B}_{n n}
\end{array}\right]
$$

Let $W$ be the reachable workspace, that is, the set of all positions and orientations reachable by the moving platform ([7] and [8]). Let $Q$ be the reachable joint space, that is, the set of all joint vectors reachable by actuated joints.

Definition $4 A$ working mode, noted $M f_{i}$, is the set of postures for which the sign of $\mathbf{B}_{j j}(j=1$ to $n)$ does not change and $\mathbf{B}_{j j}$ does not vanish.

$$
M f_{i}=\left\{(\mathbf{X}, \mathbf{q}) \in W \cdot Q \backslash \begin{array}{c}
\operatorname{sign}\left(\mathbf{B}_{j j}\right)=\text { constant } \\
\text { for }(j=1 \text { to } n) \\
\text { and } \operatorname{det}(\mathbf{B}) \neq 0
\end{array}\right\}
$$

Therefore, the set of working modes (Mf $=\left\{M f_{i}\right\}$, $i \in I$ ) is obtained while using all permutations of sign of each term $\mathbf{B}_{j j}$.

The Cartesian product of $W$ by $Q$ is noted $W \cdot Q$. According to the joint limit values, all working modes do not necessarily exist. Changing working mode is equivalent to changing the posture of one or several given legs. The working modes are defined in $W \cdot Q$ because the terms $\mathbf{B}_{j j}$ depend on both $\mathbf{X}$ and $\mathbf{q}$.

Theorem 1 The working modes separate inverse kinematic solutions if and only if the legs are not cuspidal (see [9]).

Proof 1 If one leg is cuspidal then this leg can make a changing posture trajectory without meeting a serial singularity. In this case no $\mathbf{B}_{j j}$ vanishes during

this trajectory. Reciprocally, if no leg is cuspidal, then the changing posture trajectory of one leg induces that some $B_{j j}$ can vanish.

In this study, the legs are not cuspidal so that the working modes allow the separation of the inverse kinematic solutions. The list of the most current noncuspidal serial chains is given in [9].

Example 1 For the robot Delta [10], a 3-dof manipulator (Fig. 3), there are 8 working modes (3 legs and 2 postures for each leg, with $2^{3}=8$ working modes). And for the Hexa robot [11], a 6-dof manipulator (Fig. (4), there are 64 working modes $\left(2^{6}=64\right)$. For these manipulators, the serial singularities occur when one or more legs are outstretched.

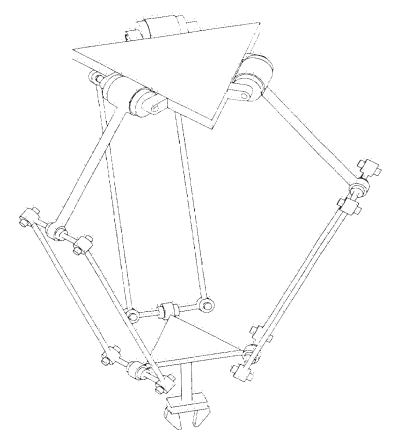

Figure 3: The Delta manipulator

\subsection{Notion of aspect for fully parallel ma- nipulators: General definition}

The notion of aspect was introduced by [1] to cope with the existence of multiple inverse kinematic solutions in serial manipulators. Recently, the notion of aspect was defined for parallel manipulators with only one inverse kinematic solution [2] to cope with the existence of multiple direct kinematic solutions.

In this section, the notion of aspect is redefined formally for fully parallel manipulators with multiple inverse and direct kinematic solutions.

Definition 5 The generalized aspects $\mathbf{A}_{i j}$ are defined as the maximal sets in $W \cdot Q$ so that

- $\mathbf{A}_{i j} \subset W \cdot Q$;

- $\mathbf{A}_{i j}$ is connected; 


$$
\text { - } \mathbf{A}_{i j}=\left\{(\mathbf{X}, \mathbf{q}) \in M f_{i} \backslash \operatorname{det}(\mathbf{A}) \neq 0\right\}
$$

In other words, the generalized aspects $\mathbf{A}_{i j}$ are the maximal singularity-free domains of the Cartesian product of the reachable workspace with the reachable joint space.

Definition 6 The projection of the generalized aspects in the workspace yields the parallel aspects $\mathbf{W} \mathbf{A}_{i j}$ so that

- $\mathbf{W A}_{i j} \subset W$;

- $\mathbf{W A}_{i j}$ is connected.

The parallel aspects are the maximal singularity-free domains in the workspace for one given working mode.

Definition 7 The projection of the generalized aspects in the joint space yields the serial aspects $\mathbf{Q} \mathbf{A}_{i j}$ so that

- $\mathbf{Q A}_{i j} \subset Q$;

- $\mathbf{Q} \mathbf{A}_{i j}$ is connected.

The serial aspects are the maximal singularity-free domains in the joint space for one given working mode.

\section{A Two-DOF Closed-Chain Manipu- lator}

For more legibility, a planar manipulator is used as illustrative example in this paper. This is a fivebar, revolute $(R)$-closed-loop linkage, as displayed in Fig. 5. The actuated joint variables are $\theta_{1}$ and $\theta_{2}$, while the Output values are the $(x, y)$ coordinates of the revolute center $P$. The passive joints will always be assumed unlimited in this study. Lengths $L_{0}, L_{1}$, $L_{2}, L_{3}$, and $L_{4}$ define the geometry of this manipulator entirely. We assume here the dimensions $L_{0}=9$, $L_{1}=8, L_{2}=5, L_{3}=5$ and $L_{4}=8$, in certain units of length that we need not specify.

\subsection{Kinematic Relations}

The velocity $\dot{\mathbf{p}}$ of point $P$, of position vector $\mathbf{p}$, can be obtained in two different forms, depending on the direction in which the loop is traversed, namely,

$$
\begin{aligned}
& \dot{\mathbf{p}}=\dot{\mathbf{c}}+\dot{\theta}_{3} \mathbf{E}(\mathbf{p}-\mathbf{c}) \\
& \dot{\mathbf{p}}=\dot{\mathbf{d}}+\dot{\theta}_{4} \mathbf{E}(\mathbf{p}-\mathbf{d}
\end{aligned}
$$

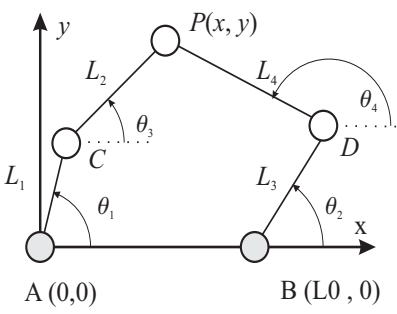

Figure 5: A two-dof closed-chain manipulator

with matrix $\mathbf{E}$ defined as

$$
\mathbf{E}=\left[\begin{array}{cc}
0 & -1 \\
1 & 0
\end{array}\right]
$$

and $\mathbf{c}$ and $\mathbf{d}$ denoting the position vectors, in the frame indicated in Fig. 局 of points $C$ and $D$, respectively. Furthermore, note that $\dot{\mathbf{c}}$ and $\dot{\mathbf{d}}$ are given by

$$
\dot{\mathbf{c}}=\dot{\theta}_{1} \mathbf{E} \mathbf{c}, \quad \dot{\mathbf{d}}=\dot{\theta}_{2} \mathbf{E}(\mathbf{d}-\mathbf{b})
$$

We would like to eliminate the two idle joint rates $\dot{\theta}_{3}$ and $\dot{\theta}_{4}$ from eqs.(5a) and (5b), which we do upon dotmultiplying the former by $\mathbf{p}-\mathbf{c}$ and the latter by $\mathbf{p}-\mathbf{d}$, thus obtaining

$$
\begin{aligned}
(\mathbf{p}-\mathbf{c})^{T} \dot{\mathbf{p}} & =(\mathbf{p}-\mathbf{c})^{T} \dot{\mathbf{c}} \\
(\mathbf{p}-\mathbf{d})^{T} \dot{\mathbf{p}} & =(\mathbf{p}-\mathbf{d})^{T} \dot{\mathbf{d}}
\end{aligned}
$$

Equations (6a) and (6b) can now be cast in vector form, namely,

$$
\mathbf{A} \dot{\mathbf{p}}=\mathbf{B} \dot{\boldsymbol{\theta}}
$$

with $\dot{\boldsymbol{\theta}}$ defined as the vector of actuated joint rates, of components $\dot{\theta}_{1}$ and $\dot{\theta}_{2}$. Moreover $\mathbf{A}$ and $\mathbf{B}$ are, respectively, the direct-kinematics and the inversekinematics matrices of the manipulator, defined as

$$
\mathbf{A}=\left[\begin{array}{c}
(\mathbf{p}-\mathbf{c})^{T} \\
(\mathbf{p}-\mathbf{d})^{T}
\end{array}\right]
$$

and

$$
\mathbf{B}=\left[\begin{array}{cc}
L_{1} L_{2} \sin \left(\theta_{3}-\theta_{1}\right) & 0 \\
0 & L_{3} L_{4} \sin \left(\theta_{4}-\theta_{2}\right)
\end{array}\right]
$$

\subsection{Parallel singularities}

For the manipulator studied, the parallel singularities occur whenever the points $C, D$, and $P$ are aligned (6). Manipulator postures whereby $\theta_{3}-\theta_{4}=k \pi$ denote a singular matrix $\mathbf{A}$, and hence, define the boundary of the Joint space of the manipulator. 


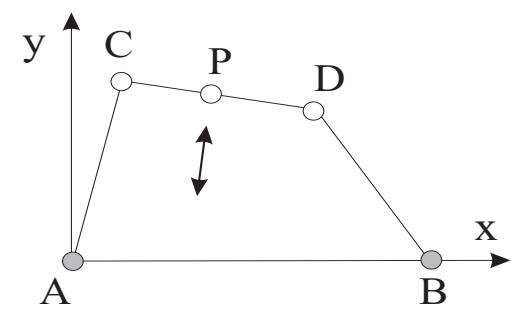

Figure 6: Example of parallel singularity

\subsection{Serial singularities}

For the manipulator at hand, the serial singularity occur whenever the points $A, C$, and $P$ or the points $B, D$, and $P$ are aligned (7). Manipulator postures whereby $\theta_{3}-\theta_{1}=k \pi$ or $\theta_{4}-\theta_{2}=k \pi$ denote a singular matrix $\mathbf{B}$, and hence, define the boundary of the Cartesian workspace of the manipulator.

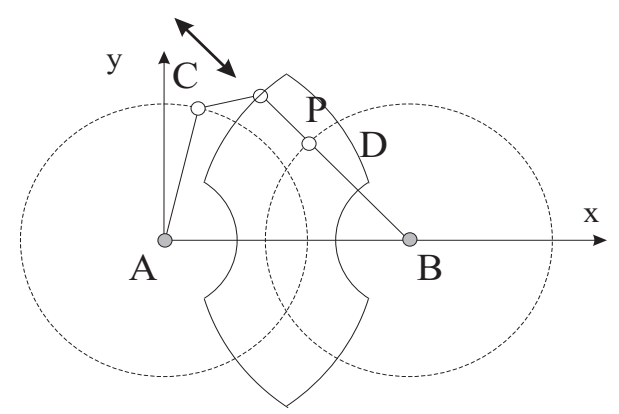

Figure 7: Example of serial singularity

\subsection{The Working Mode}

The manipulator under study has a diagonal inverse-kinematics matrix $\mathbf{B}$, as shown in eq. (7c). There are four working modes, as depicted in Fig. 8. The different working modes in the Cartesian workspace and in the Joint space are displayed in figures 9, 10, 11 and 12 .

\subsection{The generalized aspects}

For the manipulator at hand, the generalized aspects are defined with the definition 5. Figures 13 20 depict the different serial and parallel aspects obtained. Table 11 shows that there are 10 serial/parallel aspects for the manipulator ( $\mathrm{N}$ and $\mathrm{P}$ stand for negative and positif, respectively).
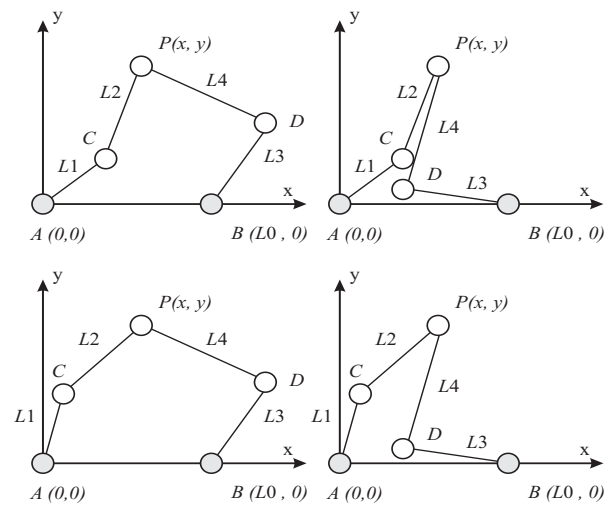

Figure 8: The four working modes
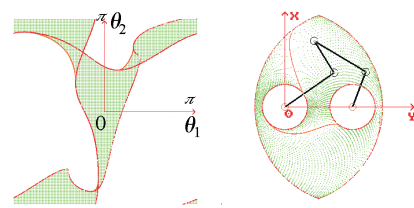

Figure 9: $M f_{1}$
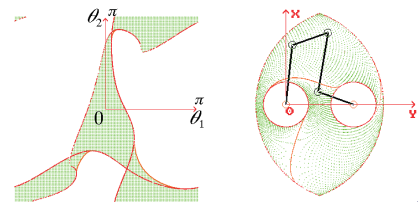

Figure 11: $M f_{3}$
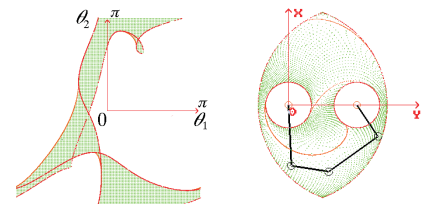

Figure 10: $M f_{2}$
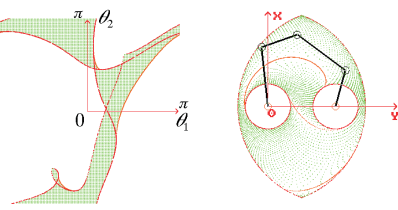

Figure 12: $M f_{4}$
In opposite to the aspects defined by [1] or by [2], the generalized aspects are not disjoint. Only, the aspects belonging to the same working mode are disjoint. In the sample in which the manipulator has only one inverse kinematic solution, the notion of generalized aspect is equivalent to the notion of aspect given by [2]. Indeed, if the manipulator has only one working mode (like the 2-RPR planar manipulator), then the parallel aspects are disjoint and represent the maximal singularity-free domain in the Cartesian workspace.

\section{Conclusions}

In this paper, the notion of aspect was defined for parallel manipulators with multiple inverse and direct kinematic solutions. The working modes were introduced to define this notion. For one working mode, we can find out the maximal singularity-free domains of the Cartesian product of the workspace with the joint 


\begin{tabular}{|c|c|c|c|c|c|c|c|c|}
\hline Figures & 13 & 14 & 15 & 16 & 17 & 18 & 19 & 20 \\
\hline $\operatorname{det}(\mathbf{A})$ & $\mathrm{P}$ & $\mathrm{P}$ & $\mathrm{P}$ & $\mathrm{P}$ & $\mathrm{N}$ & $\mathrm{N}$ & $\mathrm{N}$ & $\mathrm{N}$ \\
\hline $\mathbf{B}_{11}$ & $\mathrm{P}$ & $\mathrm{P}$ & $\mathrm{N}$ & $\mathrm{N}$ & $\mathrm{P}$ & $\mathrm{P}$ & $\mathrm{N}$ & $\mathrm{N}$ \\
\hline $\mathbf{B}_{22}$ & $\mathrm{P}$ & $\mathrm{N}$ & $\mathrm{N}$ & $\mathrm{P}$ & $\mathrm{N}$ & $\mathrm{P}$ & $\mathrm{P}$ & $\mathrm{N}$ \\
\hline $\begin{array}{c}\text { Nb of } \\
\text { generalized } \\
\text { aspects }\end{array}$ & 1 & 1 & 1 & 2 & 1 & 2 & 1 & 1 \\
\hline
\end{tabular}

Table 1: The generalized aspects

space. This work brings material to further investigations like trajectory planning and kinematic design which are the subject of current research work from the authors.

The generalized aspect are not the uniqueness domain in any case as it was shown in [2]. So, in a future study, the authors will define uniqueness domains for general fully parallel manipulators. In such domains, there are only one inverse and direct kinematic solution. These domains are of interest for the control of manipulator.

\section{References}

[1] Borrel, P. "A study of manipulator inverse kinematic solutions with application to trajectory planning and workspace determination" Proceeding IEEE International Conference on Robotic And Automation, pp 1180-1185, 1986.

[2] Wenger, Ph. and Chablat, D. "Uniqueness Domains in the Workspace of Parallel Manipulators" IFAC-SYROCO, Vol. 2, pp 431-436, 3-5 Sept., 1997, Nantes.

[3] Merlet J-P. "Les robots parallèles" HERMES, seconde édition, Paris, 1997.

[4] Stewart, D. "A platform with 6 degree of freedom" Proc. of the Institution of mechanical engineers, 180 (Part 1,15):371-386, 1965.

[5] Angeles, J. "Fundamentals of Robotic Mechanical Systems" SPRINGER 97.

[6] Gosselin, C. and Angeles, J. "Singularity analysis of closed-loop kinematic chains" IEEE Transactions On Robotics And Automation, Vol. 6, No. 3, June 1990.

[7] Kumar V. "Characterization of workspaces of parallel manipulators" ASME J. Mechanical Design, Vol. 114, pp 368-375, 1992.

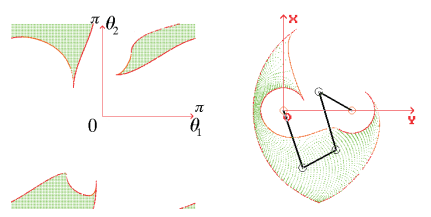

Figure 13: Aspect 1

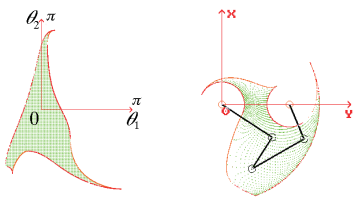

Figure 15: Aspect 3

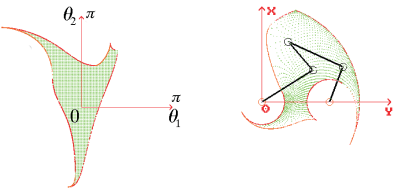

Figure 17: Aspect 6
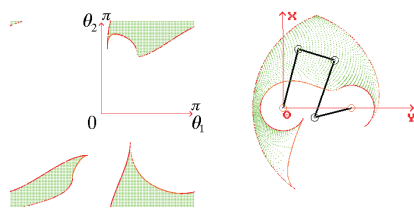

Figure 19: Aspect 9
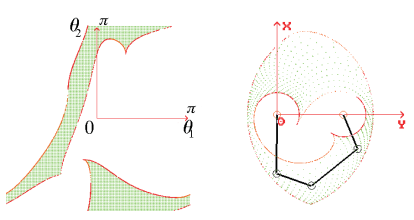

Figure 14: Aspects 2
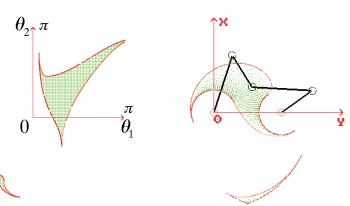

2

Figure 16: Aspects 4 and 5
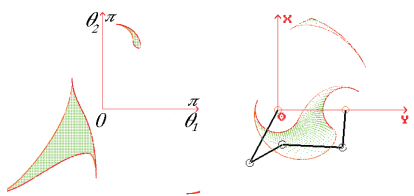

Figure 18: Aspects 7 and 8
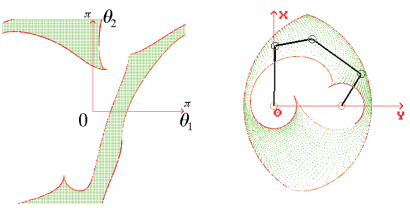

Figure 20: Aspect 10
[8] Pennock, G.R. and Kassner, D.J. "The workspace of a general geometry planar three-degree-offreedom platform-type manipulator" ASME J. Mechanical Design, Vol. 115, pp 269-276, 1993.

[9] Wenger, Ph. "A classification of Manipulator Geometries Based on Singularity Avoidance Ability" ICAR'93, pp 649-654, Tokyo, Japan, Nov. 1993.

[10] Clavel, R. "A fast robot with parallel geometry" In 18th International Symposium on Industrial Robot, pages 91-100, Lausanne, 26-28 Avril 1988.

[11] Pierrot, F. "Robot Pleinement Parallèles Légers : Conception, Modélisation et Commande" Doctorat thesis, 1991, Montpellier. 